\title{
Germanica
}

\section{«Kollektivneurose moderner Männer»}

Die Neue Sachlichkeit als Symptom des männlichen Identitätsverlusts sozialpsychologische Aspekte einer literarischen Strömung

La Nouvelle Objectivité, une névrose masculine

\section{Ulrike Baureithel}

\section{OpenEdition}

\section{Journals}

Édition électronique

URL : http://journals.openedition.org/germanica/2387

DOI : 10.4000/germanica.2387

ISSN : 2107-0784

\section{Éditeur}

Université de Lille

Édition imprimée

Date de publication : 31 décembre 1991

Pagination : 123-143

ISSN : 0984-2632

\section{Référence électronique}

Ulrike Baureithel, « «Kollektivneurose moderner Männer» », Germanica [Online], 9 | 1991, Online erschienen am: 15 Juli 2014, abgerufen am 06 Oktober 2020. URL : http://journals.openedition.org/ germanica/2387 ; DOI : https://doi.org/10.4000/germanica.2387

Ce document a été généré automatiquement le 6 octobre 2020.

(C) Tous droits réservés 


\title{
«Kollektivneurose moderner Männer»
}

\author{
Die Neue Sachlichkeit als Symptom des männlichen Identitätsverlusts - \\ sozialpsychologische Aspekte einer literarischen Strömung
}

La Nouvelle Objectivité, une névrose masculine

\section{Ulrike Baureithel}

\begin{abstract}
Nach dem Kriege war in Deutschland vielleicht noch mehr als in anderen Ländern eine Entwicklung eingetreten, bei der die Autorität des Vaters und die alte bürgerliche Moral schwer erschüttert wurde (...). Der Zerfall der alten gesellschaftlichen Autoritätssymbole wie Monarchie und Staat beeinflußte auch die Rolle der individuellen Autoritäten, nämlich der Eltern ${ }^{1}$.
\end{abstract}

1 Diese von Erich Fromm 1941 unter dem Eindruck des Nationalsozialismus formulierte Analyse, die das offenbar gewordene Autoritätssyndrom der Deutschen unter sozialpsychologischen Gesichtspunkten zu fassen versuchte, folgt in ihren Hauptlinien einer bereits Anfang 1919 in der Wiener Psychoanalytischen Vereinigung zirkulierenden Abhandlung von Paul Federn über die «vaterlose Gesellschaft». Ihr verdanke sich nämlich, so Federn, der revolutionäre Impuls von 1918, denn mit dem «Sturz des Kaisers, der Menschen und Land verlor und jetzt keine Sicherheit mehr bieten konnte, war der an eine vaterähnliche Autorität gebundene Obrigkeitskeitsstaat diskreditiert, blieben die "vaterlosen Gesellen» mit ihrem «Mutterland in innerer Verwirrtheit» zurück ${ }^{2}$. Seine von den Erfahrungen des Faschismus noch unbeschwerte, optimistische Prognose eines möglichen antihierarchischen Söhne-Bündnisses, das sich in der Kultursphäre expressionistisch artikulierte, zeigte sich allerdings hellsichtig gegenüber dem gesellschaftlichen Explosivstoff, den die Masse der vaterlosen Söhne, «die nur auf eine geeignete, neu auftretende Persönlichkeit warte(t), die ihrem Vaterideale entspricht $»^{3}$, der jungen Republik ins Haus stellte.

2 Nun hat bekanntlich die Inflation dem in Aussicht gestellten Projekt ein jähes Ende bereitet und die Söhne als ökonomisch liquidierte Monaden in den nicht endenwollenden Kampf um die nackte Existenz geworfen. Wenn jedoch die Weimarer Publizistik jedweder Provenienz ab Mitte der Zwanziger Jahre in einen unüberhörbaren 
Chorus über den Sinnverlust der männlichen Existenz verfällt, dann lohnte es möglicherweise, jenseits der Federn verpflichtenden psychoanalytischen Kategorien, nach den bewegenden Motiven solchen Gleichklangs zu fragen, denn nicht erst Adorno und Horkheimer haben im Kulturindustriekapitel der Dialektik die «Selbstverhöhnung des Mannes, der zum Subjekt, Unternehmer, Eigentümer nicht werden kann, konstatiert $^{4}$. Der patriarchale Wertezerfall durch Krieg und Inflation prägt als hervorstechendes Thema den Leit diskur s jener Jahre und verdient genauere Beachtung, insbesondere, wenn von einer Literaturströmung die Rede sein soll, die die «männliche Sache» als Markenzeichen auf ihre Fahnen schrieb und als Emblem einer neutral firmierenden Öffentlichkeit zur Verfügung stellte.

3 Schon in den frühen Zwanziger Jahren, aber verstärkt, nachdem die Wirkungen der Inflation schon absehbarer wurden und die Lebenschancen breiter Gesellschaftsschichten unterminierten, signalisiert die Klage überdie «Generalpleite der männlichen Verdienstkraft» ${ }^{5}$ die männlichen Impotenzängste. Glaubte sich Wilhelm Michel 1922 noch in der «Weltbühne» über die «neudeutsche Frau» als Kreuzung «zwischen gackerndem Huhn und zischender Natter» lustig machen und die Deutschen auffordern zu können, ihre «Weiber zur Ordnung» zu rufen ${ }^{6}$, verlagert sich ab spätestens Mitte der Zwanziger Jahre der Tenor: die überlegen-selbstironische Geste gegenüber der «Entthronung des Mannes» ${ }^{7}$ schlägt, je nach Temperament, um in zynische Resignation oder hilflosen, aber wortreichen Protest wider die «unnatürliche Umkehrung» der Verhältnisse. 1929 von Friedrich Huebner aufgefordert, über «die Frau von morgen» Auskunft zu geben, lesen sich die abgeforderten Stellungnahmen der Literaten und Publizisten durchweg als Bankrotterklärung der Männerwelt ${ }^{8}$.

«Im Umkreise dessen, was einen Mann heute beschäftigt, gibt es nichts, was noch unbedingt wertvoll erschiene» ${ }^{9}$ teilt sich das Unbehagen an der männlichen Kultur mit; «vollkommenen Schiffbruch» hätten die Männer «in den Dingen der Menschlichkeit» erlitten ${ }^{10}$, und diese «wachsende Unfähigkeit der Männer, hält Georg von der Vring den euphorischen Hoffnungen Flakes entgegen, könne auch «nicht durch die Entwicklungsklettertour der Frau kompensiert werden $»^{11}$. Indes findet auch Hollander die männliche Wirklichkeit nicht mehr für «lebenswert, sondern «lebensgefährlich und lebensfeindlich $»^{12}$ und mutet, wie übrigens viele seiner Geschlechtsgenossen und Kollegen, der künftigen Frau die Rolle der gesellschaftlichen Stabilisatorin und Hoffnungsträgerin zu. «Der Mann wird abgedankt, wird Otto Rühle 1932 in der «Literarischen Welt» den Nieder-gang der liberalen Männlichkeit einläuten und feststellen, «daß der Mann heute weder seine Sicherung durch Arbeit noch seine Sicherung durch Kampf aufrecht erhalten kann» ${ }^{13}$. Ganz so düster mochte Heinrich E. Jacob 1929 die Situation zwar noch nicht sehen, aber auch er fragt beunruhigt nach der Daseinsberechtigung seiner männlichen Zeitgenossen: «Was ist eigentlich schon ein Mann? ${ }^{14}$, worauf Axel Eggebrecht mokant kontert: «Der Mann von heute, dieses schläfrige Tierchen»» ${ }^{15}$.

5 Um der «Kollektivneurose moderner Männer» ${ }^{16}$ zu begegnen, wurde schon 1922 der Ruf nach «wirklich männlichen Typen $»^{17}$ laut, der sich in der Blütezeit der Republik unvermindert weiterpflanzt. Aber weder der Krieg, der «jene tiefe Achtung des Mannes für den Mann (...) geschaffen hat ${ }^{18}$, noch die relativ ruhige Stabilisierungsphase weiß offenbar die «Krise der Männlichkeit in Europa» zu überwinden und, wie W.E. Süskind kurz vor der nationalsozialistischen Machtübernahme resigniert feststellen muß, «die Sehnsucht nach dem "sicheren Mann"» zu befriedigen ${ }^{19}$. 
6 Zweifellos sind die männlichen Insuffizienzängste untrennbar verknüpft mit den Modernisierungserfahrungen der Zeit, die den an Leib und Seele Gekränkten individuelle Bewältigungsstrategien abverlangten. Das dabei immer wieder bemühte Geschlechtermodell verweist auf ein Phänomen, das schon vor dem Ersten Weltkrieg an Bedeutung gewann. Schon im ausgehenden 19. Jahrhundert nämlich wurde Kulturkritik mit den Metaphern des Geschlechter konflikts betrieben, so wenn zum Beispiel von der Großstadt als der «Hure Babylon» die Rede war ${ }^{20}$. Im Laufe der Zwanziger Jahre allerdings finden auf der semantischen Ebene Verwischungen und Vermischungen statt, die es nicht mehr erlauben, diesen Diskurs in eindeutig abgrenzbare Begriffsfelder aufzuteilen, sondern die als Indiz der zunehmenden Verunsicherung traditioneller kultureller Ordnungsmuster gewertet werden müssen. Wenn bis zum Krieg das dualistisch organisierte Geschlechterprinzip offenbar noch verbindlicher Wertmaßstab gewesen war $^{21}$, aufgrund dessen sich die Welt auf ebenso einfache wie unwiderlegbare Weise «erklären» ließ, so ist in der Nach-kriegszeit ein deutlicher Orientierungsverlust festzustellen.

7 Die Dichotomie von «Kultur» und «Zivilisation» etwa, geographisch verortet in Europa und Amerika und metaphorisiert in den Begriffen von «männlich» und «weiblich, diente bis in die Zwanziger Jahre hinein als kategoriales System der Weltinterpretation. 1923 noch schrieb Sieburg in der «Weltbühne» mit einem komischen Seufzer von der «Nutte» als dem «Schicksal der deutschen Gesamtheit»² und warnt vor einer Verweiblichung des Zeitalters. Und 1924 denunziert das «TageBuch» die deutschen Zustände als «weiblich, verworren und hysterisch» und verpflichtet die Deutschen als erste der Europäer, sich «wieder auf die Männlichkeit $(\mathrm{zu})$ besinnen»" ${ }^{23}$. Im Mittelpunkt des Interesses steht dabei das «amerikanische Matriarchat» mit seiner «Vergirlung der Welt». Aber schon Mitte der Zwanziger Jahre lassen sich auch Stimmen vernehmen, die den Zivilisationsprozeß und seine politische Repräsentationsform, die bürgerliche Demokratie, als männliche Leistung reklamieren, so beispielsweise in einem Plädoyer Arnold Hahns 1924:

Ich bin Ketzer genug, daß für mich die Zivilisation den freieren und stolzeren Zustand des Menschengeschlechts bedeutet; sie ist der Zustand der voraussetzungslosen Menschlichkeit, einer hochmütigen, autoritätslosen Menschheit, die es verschmäht, hinter den Röcken einer bemutternden Kultur Schutz und Führung zu suchen. (...) die Zivilisation streckt ihre Wurzeln in die klare Nährlösung des Verstandes. Und für jede Kultur kommt der Augenblick, in dem ihre schöne, aber lockere und gebrechliche Blüte vom derben Stocke des Verstandes unbarmherzig geköpft wird ${ }^{24}$.

Der Mann, der da die (weibliche Kultur-) Rose bricht, wählt seine Bilder mit Bedacht: er führt seine Rede nämlich über die Möglichkeit einer «zivilisierten Sexualität, die er sich - verräterisch genug - offenbar nur als kriegerische Begegnung vorstellen kann: «Das Faustische der Zivilisation in die Sexualität» ${ }^{25}$ hineintragen, schlägt er vor. Seine Gewaltphantasien reihen sich ein in die Phalanx derer, die das Verhältnis zwischen den Geschlechtern nur noch als Kriegsschauplatz zu entwerfen vermögen: die «Konvertierung der Erlebnisse aus dem Männerkrieg in den Bereich zwischen Mann und Frau ${ }^{26}$, wie es einmal ein Bronnen-Rezensent ausdrückte. Ob es sich allerdings bei den semantischen Verschiebungen lediglich um Umdeutungs- und Umwertungsmanöver handelt, die die Zeitgenossen von den angsterzeugenden Zumutungen der Modernisierung entlasten sollten, wie Helmut Lethen annimmt ${ }^{27}$, bleibt fraglich. Nimmt man die Aussagen, die dem «männlichen Prinzip» den 
Untergang prophezeiten, als Ausdruck eines allgemeinen Identitätsverlusts einmal ernst, so erscheint die Identifikation von «Mann» und «Sache, die die neusachlichen Vertreter ab Mitte der Zwanziger Jahre in den Diskurs einführen, nicht nur als rhetorisches «Einverständnis» mit dem Projekt der Moderne, durch das der eine «Pol» durch den anderen «aufgewertet» würde. Hatte sich der expressionistische Jünglingsaufstand der literarischen Avantgarde ${ }^{28}$ gegen die Vätergeneration gerichtet, so traten die nunmehr gealterten Söhne keineswegs deren Erbe an, für das sie auch weiterhin die Verantwortung ablehnten.

9 Für das «Männer-Pathos» der Neuen Sachlichkeit hatte Pinthus, der eine wichtige kontinuitätsstiftende Instanz zwischen beiden Strömungen darstellt, das Signal gegeben: «Nicht auf das Jünglingstum, auf das Mannwerden oder Mannsein kommt es an $»^{29}$. Der neusachliche Männlichkeitskult knüpft aber nicht einfach an das diskreditierte autoritäts- und personenfixierte Männlichkeits-Ideal des Wilhelminischen Zeitalters - den «Mimen ansprechender und repräsentativer Männlichkeit» ${ }^{30}$ - an; seine Stilisierung erfolgte vielmehr mittels eines Prinzips, das es erlaubte, sich der kompromittierenden Geschichte des Geschlechts zu entledigen und das der Strömung den Namen gab: der Sachlichkeit. Die «männliche Literatur» der Neuen Sachlichkeit sollte wie jener «Vakuumcleaner, den die Revolutionäre von 1919 beschworen, «den muffigen Müll (aus) der schmutzverstopften Epoche saugen» ${ }^{31}$ und die «betonte Herzenskälte» ${ }^{32}$ in einer «männlich tatkräftigen Zeit» ${ }^{33}$ kultivieren. Die Abhärtung der Männerkörper und -seelen, die schon Henry Ford in der Stahl-natur des Ingenieurs gegenüber den «verweichlichenden» Tendenzen der Zeit anempfohlen hatte ${ }^{34}$, war schließlich nicht nur Attitüde einer funktionslos gewordenen Intelligenz ${ }^{35}$, sondern fand ihre Entsprechung im materialisierten Produkt des Männerkollektivs: der Technik.

10 Häufig genug ist der Objekt- und Objektivitätsetischismus der Neuen Sachlichkeit unter politischen und kunstimmanenten Fragestellungen beschrieben und kritisiert worden; daß dabei der offensichtliche Zusammenhang zwischen dem eingeforderten Sachlichkeits-Postulat und seiner identitätsstiftenden Funktion für einen neuen Männlichkeitsentwurf nie in den Blick fiel, dürfte auf die Betriebsblindheit der Fragesteller zurückzuführen sein. Denn von den Zeitgenossen selbst wurde diese Verbindung immer wieder hergestellt: nicht nur Pinthus tituliert seinen richtungsweisenden Beitrag zur Neuen Sachlichkeit programmatisch mit «Männliche Literatur, auch Günther Müller spricht in seinem im gleichen Jahr erschienenen Aufsatz von den «Männern der Sachlichkeit»" ${ }^{36}$, und Utitz erkannte in der neuen Strömung «eine kraftvoll sich regende Männlichkeit, deren «ruhige(r), gehaltene(r), zähe(r) Atem reifer Menschheit in Strenge und Disziplin, Zucht und Ordnung» befähigt sei, die Krise zu überwinden ${ }^{37}$. Die «teilnahmslose Sachlichkeit, so neutral und «kalt» sie auch daherkommen mochte, war also alles andere als «uninteressiert». Mochten sich zwar «die Tatsachen ganz ohne Gefühlsverbrämung, bei völliger Ausschaltung des eigenen Ich, seiner Freuden, Schmerzen oder Ansichten ${ }^{38}$ von den Anfechtungen individueller Leidenschaften reinigen, der historischen Bürde der Gattung aber war nur mühsam zu entkommen.

11 Wenn also «die Sache» prädestiniert schien, von der Weltblamage des männlichen Prinzips abzulenken und der gestrandeten Männergeneration vorläufigen Halt zu verschaffen, um es als Maßstab doch gleichzeitig wieder unverdächtig in seine Rechte zu setzen ${ }^{39}$, so ist es um so erstaunlicher, daß in der neusachlichen Literatur häufig 
weibliche Protagonistinnen aufgefordert waren, dieses sachliche Prinzip zu vertreten. Offenbar hegten die neusachlichen Kunstproduzenten ein tiefes Mißtrauen gegenüber männlichen «Helden, und in der Tat fand, wie die neusachliche Endphase belegt, das heroische männlich-sachliche Prinzip in den unglücklichen Auftritten des bankrotten, schutzbedürftigen Pinneberg, des schwimmuntüchtigen Fabian, des tatscheustolpernden Ginster und wie sie alle heißen mögen, eine reichlich unwürdige Vertretung. "Offenbar ist die Frau besser geeignet, eine Sache vorzustellen als der Mann, vermutete Alfred Polgar schon 1926 in einer Zeitschrift, die sich in den Zwanziger Jahren um die Entwicklung modernisierter Weiblichkeit besonders verdient machte ${ }^{40}$. «Die Generation ohne Männer, so der Titel eines um 1932 veröffentlichten Pamphlets des Antifeminismus ${ }^{41}$, bemächtigte sich der Heldin, weil die verfügbaren Anti-Helden den Dienst versagten und sich der an sie gestellten Forderungen verweigerten. Ob es sich dabei nur um eine «antiemanzipatorischen Affekten» geschuldete Umkehrung der Verhältnisse handelte ${ }^{42}$, oder ob sich im Griff nach der Protagonistin über die ideologischen Vorurteile hinaus auch das bürgerlich-männliche Krisenbewußtsein, das das erodierte Geschlechterverhältnis als gesellschaftliche Bedrohung wahrnahm, abzeichnet, soll im folgenden an zwei Texten von Lion Feuchtwanger und Arnolt Bronnen verfolgt werden ${ }^{43}$.

Gewählt wurden die beiden Texte, die in der neusachlichen Forschung bisher weitgehend unberücksichtigt blieben, zum einen, weil die beiden Autoren politisch unterschiedlichen «Lagern» angehörten ${ }^{44}$ und gezeigt werden kann, wie identisch sich die männliche «Wunschproduktion, unabhängig vom politischen Standort, entwickelte. Zum zweiten stammen beide Werke aus der «heroischen» Phase der Neuen Sachlichkeit ${ }^{45}$, und schließlich siedeln beide Autoren das Geschehen in oder in allegorischer Nähe von Amerika an, was oberflächlich betrachtet die AmerikaBegeisterung des zeitgenössischen Publikums bedient haben mag, aber, wie zu zeigen sein wird, auch als Negativfolie für die Weimarer Realität diente. Ausschlaggebend jedoch war im Rahmen meiner Fragestellung der Umstand, daß in beiden Texten weibliche Hauptfiguren in einem vordergründig symmetrisch umgekehrten Geschlechterarrangement die Herausforderungen und Auswirkungen sachlicher Herrschaft auf den «neuen Menschen» (Bronnen) ausloten sollen.

Feuchtwangers «Petroleuminseln» führt auf experimentell verkleinertem Raum den Kampf kapitalistischer Gegenwart gegen ein feudal-koloniales Gestern vor. Personifiziert wird der abstoßende, aber durch die ölförderung rentable Teil der Inseln durch die häßliche Deborah Gray, die als omnipotente Herrscherin das Prinzip Sachlichkeit vertritt: «sehr häßlich, vom Öle lebend, Überfluß schöpfend aus den Mängeln des Staates, kalt vor den Genüssen des Klassenkampfes, ohne Interesse an Gott» (P 56). Ihre Herrschaft, die sich an «der kalten Welt der Zahl» orientiert, kollidiert unversöhnlich mit der Widersacherin Grays, der schönen Charmian Peruchacha, die durch ihre sinnlichgenußsüchtige Lebensweise in spätfeudaler Manier den Ablauf des Petroleumgeschäfts gefährdet. Sie tritt gegenüber der unsentimentalen, zu zweckrationaler Endkontrolle neigenden Gray, die weiß, daß «ein Mann ein Mann und ein Geschäft ein Geschäft» (P 25) ist, als «erstklassige Erscheinung, die die Männer der Inseln in ihrem Bann hält, auf. Signiert Gray für den Produktionsbetrieb auf den Inseln, sorgt Peruchacha für die Reproduktion, insbesondere ihrer männlichen Bewohner. «Männliches Geschäft» und «feminine Störungen» werden als dualistische 
und in unversöhnbarem Streit verwickelte Praxen vorgestellt. Der Kampf der beiden Frauen, der auf dem Kriegsschauplatz der sexuellen Leidenschaften ausgetragen wird, entzündet sich im Zusammenhang mit einer ölkrise, die rücksichtslos rationalsachliches Kalkül der Ölmagnatin notwendig macht ${ }^{46}$. Bindeglied zwischen beiden Sphären ist der typische amerikanische «poker-face-man» Ingram: «energisch, gesinnungslos, genußsüchtig, zynisch, sehr fähig» (P 57), weiß sein Steckbrief. Er versucht die ölkrise auszunutzen, um mit Gray Geschäfte zu machen und gerät mit ihr in Konflikt, als offenbar wird, daß er sich zur Peruchacha hingezogen fühlt. Feuchtwanger selbst hat in der «Selbstanzeige» des Stückes 1927 darauf verwiesen, es sei ihm um «den Kampf einer starken, begabten Frau (...) gegen das häßliche Gesicht, das ihr auf ihren Weg mitgegeben ist $\rangle^{47}$, gegangen. Auf diesen Hinweis hin hat die Forschung immer wieder den Zusammenhang mit dem im 14. Jahrhundert spielenden «Maultasch»-Roman hergestellt und dabei die unübersehbaren Differenzen beider Texte ignoriert ${ }^{48}$. Die Interpretationsvorlagen insistieren in der Regel auf der «Auseinandersetzung mit dem Ästhetizismus» der Zeit ${ }^{49}$ oder den für Feuchtwanger typischen Kampf des Häßlichen mit dem Schönen $»^{50}$ und vernachlässigen völlig die Bedeutung, die der Geschlechtertausch im Stück haben könnte. Einen Schlüssel für ein neues Verständnis des Stückes könnte das von Feuchtwanger zentral angelegte Gleichnis, das er Lelio, dem in Ungnade gefallenen Leiter der Abteilung 17, in den Mund legt, liefern:

In Ohio wurde vor zwei Jahren folgender Versuch mit Ratten gemacht. Man trennte sechs Männchen durch eine elektrische Platte auf der einen Seite vom Futter, auf der anderen Seite vom Weibchen. Nach 72 Stunden Hunger und Geschlechtsentbehrung drängten fünf Männchen zum Futter und eines zum Weibchen. (...) Als man das Experiment umkehrte und mit sechs Weibchen wiederholte, ging ein Weibchen zum Futter und fünf zum Männchen (P 41f).

Das in neusachlicher Mode behaviouristisch angeordnete Experiment veranschaulicht das sozial-psychologische Modell des Stückes: unter Versuchsbedingungen soll sich zeigen, ob Deborah Gray im Zweifelsfall den Weg der fünf Weibchen geht und ihrer Schwäche für Ingram nachgibt, oder den der sechsten Rättin und rücksichtslos das Geschäftsinteresse vertritt. Die Frage nach einer Synthese beider Möglichkeiten - «(...) da man ein Mensch ist und keine Ratte, müßte es eine Möglichkeit geben, den Weg der fünf mit dem Weg der einen zu verbinden» (P 94) - wird im Stück negativ entschieden, denn Deborah bringt Petroleum und Liebe gerade nicht «unter einen Deckel». Zwar siegt sie über ihre Gegnerin, durchkreuzt Ingrams Manöver und erobert die uneingeschränkte Kontrolle über die Inseln - aber nur, indem sie alle Imponderabilien aus ihren Überlegungen ausblendet.

Der umständelose Analogieschluß zwischen dem behaviouristischen Modell und anthropologischer Disposition ist indes problematisch, denn er suggeriert, daß die «Gleichheit» zwischen Ratten und Rättinnen auch zwischen Männern und Frauen existiert. Aber Gray ist aufgrund ihrer Häßlichkeit auf dem traditionellen Heiratsmarkt, auf dem die ungleiche Macht Verteilung zwischen den Geschlechtern sozial ausbalanciert wird, nichts wert, und so ist ihre Entscheidung für die ölkarriere keine autonome, sondern bleibt immer an die strukturellen Asymmetrien im Geschlechterverhältnis gebunden. Gray, die grotesk überzeichnete «Kälte-Maschine» der Neuen Sachlichkeit, vertritt die im Stück eindeutig «männlich» identifizierte Sachlichkeit, sie wird aber von ihrem Geschlecht immer wieder eingeholt. Das Prinzip Sachlichkeit geht in der Herrschaft der Dinge eben nicht auf: Gray handelt wie ein 
Mann - aber sie ist kein Mann. Das Gesetz der Inseln, das sie vertritt, ist nicht das ihre, ihr Status ist ein geliehener, und das macht sie angreifbar, aber auch überlegen.

Sinnfällig wird dieser Zusammenhang im Stück in der metaphorischen Verklammerung von Geschäft und Sexualität: die Bohrtürme, die den Reichtum der Inseln garantieren, das Strohhutstechen im Park der Peruchacha, die getrockneten Bananen, die auf den Inseln als Synonym für die Wertlosigkeit einer Sache gebraucht werden, stehen zweifelsfrei für die phallische Ordnung auf den Inseln. «Potente Burschen müssen potent bezahlt werden» (P 45), spielt Ingram seinen «Heimvorteil» gegenüber Gray aus, und die Inselbewohner wissen ihr «Lotterielos im Portefeuille, weil auf «den Braunen Inseln ein jeder den Marschallstab in der Hose trägt» (P 64). Ökonomische und sexuelle Potenz gehen eine unverbrüchliche Symbiose ein ${ }^{51}$, und dort, wo Ingram geschäftlich unterliegt, setzt er seine «männlichen Qualitäten» (P 61) ein, um Gray zu übervorteilen. Dieses durch das gesamte Stück unterschwellig thematisierte Gewaltverhältnis - und darum handelt es sich bei Ungleichen - ist nicht umkehr-bar, und Ingram, «der Kraftprotz mit Stiereitelkeit, unterliegt schließlich nur, weil Gray ihm gegenüber im entscheidenden Augenblick im Vorteil ist, sie bedarf seiner nicht als Reproduktionsinstanz. «Wenn sie (die Männer/UB) geil sind, werden sie sentimental» (P 106) konstatiert sie und kommt in bezug auf Ingram zum Ergebnis, daß «Preis und Ware nicht im rechten Verhältnis stehen» (P 86). Ihre Fähigkeit $z u$ vergessen und $z u$ trennen lassen sie den ungleichen Kampf schließlich gewinnen: «Hirte Israel, Debsy, sie haben die Technik» (P 108), attestiert ihr der Colonel. Das einverständige, gleichwohl aber melancholische Plädoyer Feuchtwangers für die kapitalistische Gleichzeitigkeit «die Antwort gibt Amerika» (P 107) - legt er der Gray in den Mund: «Ich wollte, Sie hätten mich nicht gezwungen, dieses erstklassige Geschäft zu machen» (P 93). Ihre Trauer um den Verlust aller Bindungen setzt sich aber deutlich ab von der rückwärtsgewandten kulturkritischen Klage um die verlorenen Paradiese der Vormoderne. Mit dieser Haltung trifft sich Feuchtwanger in mancher Hinsicht mit Arnolt Bronnen, der sich ebenfalls des Projektionsfeldes Amerika bedient, um das als destabilisiert empfundene Geschlechterarrangement im eigenen Land zu verarbeiten ${ }^{52}$. In der fremden Kultur, inkarniert im Feindbild des kulturlosen «amerikanischen Matriarchats, finden die eingangs beschriebenen Impotenzängste ihr extremes Zerrbild, vor dem sich die Stilisierung der neusachlichen Männlichkeit um so wirkungsvoller abhebt. Die Adaption des amerikanischen «Geschlechterkriegs» beschränkt sich dabei gerade nicht nur auf die konservativen Kulturkritiker, sondern bedient auch jene, die die amerikanische Zivilisation gerne auf europäische Verhältnisse übertragen gesehen hätten.

18 Arnolt Bronnen jedenfalls siedelt seinen Roman ebenfalls im Amerika der Vorkriegszeit an; er protokolliert Lebens-Film und Film-Leben der Schauspielerin Barbara la Marr, die als unbekannte Reatha Watson in der Film-Metropole Hollywood Karriere machen will. Ihr Aufstieg führt dabei über wechselnde Männer, die sie, um nicht selbst durch sie ruiniert zu werden, mit «männlichen» Methoden bekämpft. Ähnlich wie Feuchtwanger ist auch Bronnen dem traditionellen Geschlechterdualismus verpflichtet: er verlegt Reathas Ursprung in die «wilden Wälder Washingtons, Reatha ist nichts als «Natur, aus Natur geboren und mit ihr identisch. Anders aber als Feucht wanger, der die ontologisierten «männlichen» und «weiblichen» Qualitäten auf zwei verschiedene weibliche Figuren verteilt, um sie auf ihre Resistenzkraft hin zu prüfen, verlegt Bronnen den Widerspruch von «männlichem Geschäft» und «femininen Störungen» in eine Figur, Barbara la Marr. Der im Roman vorgestellte Konflikt entzündet sich 
zwischen den «naturnahen» Bedürfnissen ihres Leibes einerseits und den Karrierewünschen andererseits:

Aber mitten in den Gletschern ihrer Gedanken, mitten durch die frostigen Zahlen ihres Hirns, mitten durch die unendliche Kälte ihre Himmels und ihrer Sterne fühlte sie den Mann neben sich (...). Ihr Himmel war kalt, aber um so heißer war ihre Erde (B 41). ähneln in ihrer grotesken Überzeichnung durchaus dem männlichen Personal in den «Petroleuminseln». Während dort Onkel Obadjah an seinen von der Kulturindustrie geforderten «Oden für Männer» scheitert und sich der zeitlosen «Homerübersetzung für die Jugend» verschreibt ( $\mathrm{P} 105)^{53}$, läßt Bronnen Reathas Vater («singende Säge, Häuptling der Väter von Yakima, B 95) als melancholischen Geiger auf dem Dach die Erinnerung an europäische Kulturwerte beschwören. Und Lelio, der gestrandete «Leiter der Abteilung 17» der Inselgesellschaft karikiert den ohne Arbeit um seine Identität gebrachten Mann, der Kreuzworträtsel löst und, «wenn er besonders intensiv ist, (...) einen Satz Tennis (spielt)» (P 79). Er findet in Lytell, Ainsworth oder Converse im Bronnen-Roman sein würdiges Pendant, und vergleicht man Ingram aus den «Petroleuminseln» mit Deely, sind die Parallelen so auffällig, daß man fast geneigt ist, bei Bronnen ein Plagiat zu vermuten.

21 Weshalb aber wird die neusachliche «Liebe zum Adäquaten»" ${ }^{54}$ ausgerechnet als weibliche Kompetenz vorgeführt, diese «angewandte Sachlichkeit, die Barbara la Marr angesichts des erpresserischen Selbstmordversuchs ihres einstigen Geliebten inszeniert: «unrührbar, seinen Tod trinkend» (B 247) nämlich fragt sie zum Entsetzen der anwesenden Männergesellschaft: «Warum retten?» Reatha entzieht sich durch ihre Verwandlung in Barbara la Marr ihrem Dienst an der Gattung, sie verweigert den männlichen Erlösungsauftrag «an das Weib». Versuchte Lytell, Reathas erster Mann, ihre «Natur» noch als kräftespendendes Reservoir für sich zu privatisieren, vollzieht sich im Romanverlauf die Vergesellschaftung des Weibes, diese «letzte tolle Karte» (B 172) im Männerspiel im Medium des Films: «Die Sehnsucht der Nation wird auf sie strömen» prophezeit ihr der Regisseur Fitzmaurice. «Aber wird ihr Körper offen sein, sie aufzunehmen?» (B 197) $)^{55}$.

Sind es aber tatsächlich «nur» die männlichen Verlustphantasien, die sich über das Motiv der Umkehrung in beiden Texten impliziten Ausdruck verschaffen? Oder lassen sich Hinweise auffinden, weshalb beide Autoren ihre Frauenfiguren als Überlebensfähigere imaginieren?

1929 veröffentlichte Bronnen im nationalsozialistischen «Vormarsch» einen Aufsatz über «die weibliche Kriegsgeneration $»^{56}$, in dem er die Schauspielerinnen-Vita als exemplarischen Fall für die Fähigkeit der Frauen, «den Bruch mit den morsch gewordenen Bindungen» mutig herbeizuführen, ihren «Zustand für provisorisch» zu erklären und die «Trennung zu proklamieren, anführt und ihnen andient, seine Männergeneration von der ewig kleinlichen «Sorge ums tägliche Brot zu entlasten» ${ }^{57}$. Sowohl Bronnens Tochter Barbara als auch seine Ehefrauen Hildegard und Renate berichten unabhängig voneinander von Bronnens lebenslangem Wumsch, sich frei von 
Bindungen zu fühlen, sich trennen, vergessen zu können ${ }^{58}$. Und Jaretzky sieht in seiner Würdigung der «Petroleuminseln» Grags Potenz in «ihrer Freiheit von allen historische Bindungen, von aller verpflichtenden Moral und allen Schönheitsempfindungen ${ }^{59}$. «Und in der Tat: untersucht man die beiden Texte auf diese Dimension hin, fällt auf, daß sowohl Deborah Gray als auch Reatha / Barbara sich vom Ballast der Geschichte befreit haben. Im Unterschied zu Peruchacha, der Statthalterin des kolonialen Erbes und befrachtet mit dessen Vergangenheit, tritt Deborah Gray als Geschichtslose auf die historische Bühne und übt sich lustvoll in der modernen Tugend des Vergessens. Augenblickliche Entscheidung und entscheidender Augenblick sind mit einem Denken in Kontinuitäten unvereinbar. Im einzigen Fall, wo sie gegen dieses Prinzip verstößt in ihrem privaten Rachefeldzug gegen Peruchacha - muß sie umgehend die Zinsen bezahlen.

Aber auch Barbara la Marr besitzt keine individuelle Geschichte, deren Entwicklung zu verfolgen wäre. Trotz vielfältig schlechter Erfahrungen bildet sie sich nicht und entwickelt sie sich nicht: «Gott sei Dank bin ich weder verblendet durch Erfahrung und durch Wissen» (B 94). Ihren Ursprung negierend, schlüpft sie in die Rolle der vom Pferd gefallenen Schauspielerin Marr und arrangiert ihren Namen aus den Versatzstücken der verstorbenen Vorgängerin, einem früheren Varietenamen - «Moore nannte mich die Tänzerin Barbara» (93) - und einem Präfix, das an nichts als ihr Geschlecht erinnert. Ebenso wie Gray entlastet sie sich von allen Erinnerungen: «wie lange war ich eigentlich mit Jack Lytell verheiratet?» Eben diese Traditions- und Geschichtslosigkeit faszinierte auch die zeitgenössischen Amerikareisenden, dessen Bewohnern Henry Ford ins Stammbuch schrieb: «Du sollst die Zukunft nicht fürchten und die Vergangenheit nicht ehren»" ${ }^{60}$, und für die der Keyserlingsche «Chauffeur in der Lederjacke, der keinen Ballast mit sich führt, zum Typus avancierte.

Daß eine erklärtermaßen «männliche Literatur» - «wir wollen ruhige, ernste und männliche Stücke bringen $»^{61}$ - die gegen die femininen, "geschäftsstörenden Abschweifungen» ( $\mathrm{P}$ 25) reklamierte Bindungslosigkeit ausgerechnet an weibliche Figuren delegiert, bleibt erstaunlich. Möglicherweise wog das Kulturerbe auf den männlichen Schultern doch schwerer, war die Entwurzelung des modernen Bewußtseins für seine Träger problematischer als die programmatischen Stellungnahmen des Zeitalters vermuten lassen. «Jeder Schritt vorwärts: ein sich losringen von diesem Allumfassenden mütterlichen Schöße der anfänglichen Unbewußtheit, schreibt CG. Jung $1928^{62}$, und folgt man der Bronnen-Analyse Aspetsbergers, ging es Bronnen in seiner literarischen Produktion der Weimarer Zeit darum, die «genitale Position sicher zu begründen ${ }^{63}$. Daß die Negation des Ursprungs eine notwendige neuzeitliche Leistung des männlichen Bewußtseins darstellt, hatte schon Hegel in der «Phänomenologie» ausgebreitet, und Marianne Schuller hat auf dieser Grundlage auf die widersprüchliche bürgerlich-männliche Subjektkonstitution aufmerksam gemacht:

Der Eintritt in die Ordnung des Gemeinwesens durch den Mann fordert das Vergessen, das Versinken, fordert den Tod der Erinnerung dessen, woraus der Mann hervorgegangen ist. Als Ort des Ursprungs muß die Frau, um der Erarbeitung des menschlichen / männlichen Gebots willen, vergessen werden ${ }^{64}$.

Der Mann, der den als lästig empfundenen Bindungen entfliehen will, muß sich in der Abarbeitung an der Natur als «Allgemeines» setzen und erzeugt sich doch gleichzeitig an dem, was er abzuspalten bestrebt ist. Die Erinnerung an den Ursprung erweist sich als verhängnisvoll für die männliche Identitätskonstitution, und so, wie die moderne 
Gesellschaft ihre Geschichte nur als serielle Aneinanderreihung von Momentsituationen wahrnimmt und sie ihrer historischen Potenz beraubt, so sind ihre Repräsentanten gefordert, ihre Existenz als geschichts- und erinnerungslose zu ertragen, ohne dieser Herausforderung wirklich gerecht werden zu können. Brentano, der die Marr-Figur als «neuen Helden» feiert und sie der jungen Literatengeneration ans Herz legt, würdigt Bronnen als Vertreter einer Literatur, die sich von der Last der Geschichte und der Erinnerung befreit hat:

Die literarische Produktion (der) neuen deutschen Generation (...) kommt unvermittelt und direkt aus einer fernen Zeit, an die es keine Erinnerung gibt, jäh in die Gegenwart mitten hinein. Wenige Probleme der sehr problematischen Vergangenheit belasten $\operatorname{sie}^{65}$.

«Die ontogenetische Geschichtslosigkeit und fehlende soziale Einbindung, die Carl Wege den «Männern der Sachlichkeit» als einzig einbringbares Kapital zugesteht ${ }^{66}$, wurde von den «Frauen der Sachlichkeit» offenbar «besser» investiert und verwaltet: «In den Unterschlüpfen, die sowohl die Autorinnen als auch die Heldinnen bewohnen, hinterlassen sie keine Spuren» ${ }^{67}$.

Es ist kein Zufall, daß die «neue Heldin» von Bronnen ausgerechnet als Schauspielerin eingeführt wird, er sich eine Schauspielerinnen-Vita als Vorlage für sein Experiment nimmt und darüberhinaus das Ganze als Spiel inszeniert. Es geht darum, die Möglichkeiten nicht-authentischer Lebensweise im Spiel auszutesten. Veräußerlichung wird zu Barbaras zweitem Lebensprinzip: «(...) ihre Kälte schien um so unechter, je echter sie war» (B 111) und «mein Körper ist erlogen». Sie simuliert in einem unablässigen Rollenspiel Verstellung und Mimikry als notwendige Überlebensstrategien in der modernen Gesellschaft. Geschlagen sind da die, die auf Identität bestehen, ihre Geschichte nicht zu verleugnen imstande sind und sich im Netz der bürgerlichen Tragik verwickeln. Barbara indessen, die den Konflkit zwischen der «blühenden Landschaft ihres Leibes» (B 116) und dem «Eis ihres Hirn(s)» (B 42) auszutragen hat, ist zu tragischer Verwicklung gar nicht fähig, und ihr tödliches Ende kann im Olymp der Tragik keinen Platz mehr reklamieren. Barbara la Marr spielt im zentralen Film ihres Lebens in einer Figur nacheinander die Rollen, die der männliche Weiblichkeitsentwurf für sie vorsieht: die Erlöserin und die Megäre. Aber erst, als sie aufgibt «tief zu sein, überträgt sich der Funke auf die ihr feindlich gesinnte Kritikerclique: das «Bild vom neuen Menschen, «lichtgeboren und dem Licht verbündet» (B 242) entspringt der Leere des Gefühls, in deren «bläulicher Flamme (B 249) alles erlischt: Warum retten?».

Schon die zeitgenössische Kritik hat häufig auf die dem Roman eigene Vermischung von Realität und Fiktion, die für die Zeitgenossen offenbar hohen Erklärungswert hatte, hingewiesen. Das risikoreiche Männerspiel fordert ein Leben im «als ob, das die Gefahr erträglich macht. Exterritorialisiert sind diese Existenzen, wie Ingram, der spurlos verschwindet und andernorts wieder auftaucht. Der Spielleiter in den Petroleuminseln enthebt das Personal denn auch von der Bürde tragischer Katharsis, «weil man nämlich auf der Bühne weder daran denkt, sich zermalmen zu lassen, noch sich zu erheben» ( $P$ 82).

Und trotzdem zeigen sich die weiblichen Figuren durch den ihnen angehefteten Geschlechtscharakter erfolgreicher im geforderten Maskenspiel der Moderne: Ortlos überhaupt, haben sie keinen Ort, der zu negieren, zu verlieren, zu beklagen wäre. Eben jenen Ort, auf den sich die Kultur kritik und ihre neusachlichen Kontrahenten 
unausgesprochen - nostalgisch die einen oder rigid sich abgrenzend die anderen beziehen: die gemeinsame Geschichte. Als gemeinsamer Bezugspunkt schließt er beide Fraktionen des Weimarer Diskurses zusammen, verklammert bleiben sie durch ein identisches Gefühl der Ohnmacht angesichts der Erfahrung, daß ihnen die Basis zu entgleiten droht, auf der ihre Identität sich gründete.

Die neue Grundlage auf dem Boden der «nackten Tatachen» herzustellen, wie es die «Männer der Sachlichkeit» erprobten, erwies sich indes als unsicheres Projekt. Denn die «Sachen, an die sich «zu halten, an denen «festzuhalten» aufgefordert wurde, zeigten sich unzuverlässig und offenbarten sich oft genug als Fälschung: «der Tatbestand rutschte aus und ließ sich nicht fassen, wie es Kracauer 1928 ironisch im «Ginster»-Roman formuliert ${ }^{68}$. Die Tatsachen, mit denen die neusachlichen Helden sich herumzuschlagen hatten, blieben «Sachen» ohne Tat und die erbärmlichen Täter satisfaktionsunfähig. Unter der «Tarnkappe» der «Sache» unternahm es diese Generation der Blindgänger, die es, wie Ginster, «verabscheut, ein Mann werden zu müssen ${ }^{69}$, sich von ihrer Verantwortung für die Geschichte $\mathrm{zu}$ entledigen; ausgerechnet mittels jener Sache, die das «männliche Prinzip» über seine kläglichen Auftritte in der Weltgeschichte hinwegretten sollte.

Der «entzeitete» Mann der Neuen Sachlichkeit, der sich von der Last der Entzweiung befreit, hatte allerdings nur eine kurze Premiere, denn sein weiblicher Gegenpart zeigte sich den Herausforderungen gewachsener. Daß dabei den männlichen Entwürfen nicht nur das Bewußtsein ihrer Überlegenheit eingeschrieben ist, sondern die Verpflichtung des weiblichen Personals auf die «Sache» gleichzeitig mit der Domestikation des noch nicht Unterworfenen einhergeht, signalisieren die Finale beider Texte: Gray opfert dem «adäquaten Verhalten» ihre Neigung für den Mann, Barbara la Marr endet gebändigt in Zelluloid und ermordet auf der Bühne des Lebens. Die Probe ist zum Ernstfall geworden. Das jedoch haben die Neusachlichen viel zu spät erkannt.

\section{NOTES}

1. - Erich Fromm, Die Furcht vor der Freiheit, Stuttgart, 1983, S. 187.

2. - Paul Federn, Die vaterlose Gesellschaft, Wien, 1919, S. 13.

3. - Ebda., S. 28.

4. - Max Horkheimer/Th.W. Adorno, Dialektik der Aufklärung, Frankfurt, 1971, S. 137.

5. - G. Schaetter, «Nachkriegsliebe», in Das Tage-Buch, 1924.

6. - Wilhelm Michel, «Die echte deutsche Frau», in Weltbühne 41/1922, S. $404 \mathrm{f}$.

7. - M.M. Gehrke, «Die Jagd nach der Frau», in Weltbühne 52/1924, S. 944.

8. - Friedrich M. Huebner, Die Frau von morgen wie wir sie wünschen, Leipzig, 1929.

9. - Otto Flake, «Die alte Aufgabe - die neue Form», in Huebner, S. 167.

10. - Hans Henny Jahnn, «Gesund und angenehm», in Huebner, S. 144.

11. - Georg von der Vring, "Offensive der Frau», in Huebner, S. 52.

12. - Walther von Hollander, «Autonomie der Frau», in Huebner, S. 30.

13. - Otto Rühle, «Der Mann wird abgedankt», in Literarische Welt 41/1932, S. 8. 
14. - Heinrich E. Jacob, «Haarschnitt ist noch nicht Freiheit», in Huebner, S. 130.

15. - Axel Eggebrecht, «Machen wir uns nichts vor», in Huebner, S. 122.

16. - Oscar H. Schmitz, «Die Schutzbedürftigkeit des Mannes», in Schweizerische Rundschau 10/1930, S. 788.

17. - Claude Mckay, «Three Soldiers - das neue Amerika», in Der Gegner, 1/1922, S. 14.

18. - Ernst Robert Curtius, «Zivilisation und Germanismus», in Neuer Merkur 8/1924, S. 302.

19. - W.E. Süskind, «Geist und Männlichkeit», in Die Literatur 12/1932, S. 682.

20. - Sigrun Anselm, «Emanzipation und Tradition in den Zwanziger Jahren», in Anselm/Bode(Hg.): Triumph und Scheitern der Metropole, Berlin, 1989, S. 253-74, hier: S. 256.

21. - Daß der sich mit der bürgerlichen Gesellschaft ausbildende Geschlechter kanon unterschiedlichen Bewertungen unterlag, und beispielsweise die lebenphilosophisch orientierte bürgerliche Frauenbewegung schon frühzeitig die «Aufwertung des Weiblichen» betrieb, stellte die grundsätzlich dichotomisch strukturierte Vorstellungswelt dabei nicht in Frage.

22. - Friedrich Sieburg, «Die Trilogie der fehlenden Leidenschaft», in Weltbühne 11/1922, S. 305.

23. - O.L. in Das Tage-Buch 33/1924, S. 1182.

24. - Arnold Hahn, «Die zivilisierte Sexualität», in Das Tage-Buch 45/1924, S. 1586, Hervorhebung UB. Auf das Phänomen einer voraussetzungslosen Zivilisation wird an anderer Stelle zurückzukommen sein.

25. - Ebda, S. 1589.

26. - Maxim Ziese in einer Rezension des la Marr-Romans von Arnolt Bronnen in der DAZ vom 28.6.1928; zitiert nach Arnolt Bronnen, Werke Band 3, Klagenfurt 1989, S. 374.

27. - Helmut Lethen, «Freiheit von Angst». Über einen entlastenden Aspekt der Technikmoden in den Jahrzehnten der historischen Avantgarde 1910-1930, in Großklaus/Lämmert (Hg.), Literatur in einer industriellen Kultur, Stuttgart, 1989, S. 72-98.

28. - Marlis Gerhardt weist zu Recht darauf hin, daß es auf diesem «SprachKampfplatz» tatsächlich um einen Vater-Sohn-Konflikt geht, auf dem «die Töchter nichts zu suchen haben. Sie stehen am Rande des Dramas, das sich zwischen Vätern und Söhnen abspielt und mit dem allmählichen Verschwinden der Väter endet». Marlis Gerhardt, Stimmen und Rhythmen: Weibliche Ästhetik und Avantgarde, Neuwied, 1986, S. 11.

29. - Kurt Pinthus, «Männliche Literatur», in Das Tage-Buch 10/1929.

30. - Julius Talbot, Was sind Revolutionen? (8 Teil 1), in Der Gegner 1/1919, S. 16.

31. - Ders.: Was sind Revolutionen?, in Der Gegner 2.3/1919, S. 1.

32. - Axel Eggebrecht, in Literarische Welt 1/1932, S. 6.

33. - Alfred Döblin anläßlich der Preisverleihung an junge Prosaisten 1927, in Literarische Welt 11/1927, S. 1.

34. - Henry Ford, Mein Leben und Werk, Leipzig, 1923.

35. - Vgl. hierzu: Helmut Lethen, «Kältemaschinen der Intelligenz. Attitüden der Sachlichkeit», in Ernst Wichner/Herbert Wiesener (Hg.): Industriegebiete der Intelligenz. Literatur im Neuen Berliner Westen der 20er und30er Jahre, S. 119-53, hier: S. 128.

36. - Günther Müller, «Neue Sachlichkeit in der Dichtung», in Schweizerische Rundschau, 1929, S. 712; interessant in diesem Zusammenhang ist übrigens, daß sich die «Sach-lichkeit» nicht ausschließlich an das biologische Geschlecht heftet, beide Autoren subsumieren auch Literatur aus Frauenhand unter die «männliche Literatur».

37. - Utitz, Die Überwindung des Expressionismus, Stuttgart, 1927, S. 181.

38. - Franz Matzke, Jugend bekennt: So sind wir, Leipzig, 1930, S. 69.

39. - Hier bilden übrigens der zeitgenössische Technikdiskurs und die literarische Rede eine gemeinsame Nahtstelle. Die «Form werdung» des «männlichen Prinzips» in der Technik, die etwa Krollmann mit ausgeprägt organizistischer und phallozentrischer Metaphorik in seinem Band «Schönheit der Technik» (1928) beschreibt, korrespondiert mit ihrer gleichzeitigen Ästhetisierung in der Kultur Sphäre. Mit der Bronnen eigenen Offenheit hatte schon die dem 
Expressionismus zuzuschlagende «Septembernovelle» die eindeutig phallisch konnotierte «SACHE» als novellistisches Ding-Symbol eingeführt, es aber noch weitgehend an einen individuellen Träger gebunden. Die neusachliche Sexualmetaphorik ist vermittelter, weil sich hier «die Sache» programmatisch vom individuellen Repräsentanten ablöst.

40. - Alfred Polgar, in Die Dame, 1926, S. 2.

41. - Rudolf Thiel, Die Generation ohne Männer, Berlin, 1932; der Band liest sich übrigens als Paradebeispiel der kulturellen Orientierungskategorie «Geschlecht». Freuds Triebtheorie beispielsweise gilt Thiel als Ausdruck für eine um ihre Männlichkeit gebrachte Generation, Rathenau als Vertreter jüdisch-aufgeklärten Denkens wird als «weib-lich» denunziert und Spenglers Kulturpessimismus als «unmännliches Untergängertum» gedeutet.

42. - So das Resümee Wittmanns in ihrer Analyse von Feuchtwangers «Petroleuminseln»; vgl. Livia Z. Wittmann, «Der Stein des Anstoßes, in Jahrbuch für Internationale Germanistik, 2/1982, S. 56-78.

43. - Lion Feuchtwanger, «Die Petroleuminseln», in Drei angelsächsische Stücke, Berlin, 1927. Arnolt Bronnen, Film und Leben Barbara la Marr, Berlin, 1927. Neuauflage in Arnolt Bronnen, Werke Bd. 3, Klagenfurt 1989 (Mit Zeugnissen zur Entstehung und Wirkung).

44. - Lion Feuchtwanger als der humanistischen Traditionen verpflichtete Autor hatte sich von der zeitgenössischen Sachlichkeitsbegeisterung frühzeitig distanziert; daß er jedoch maßgeblich an der literarischen Aufnahme und Verarbeitung des neusachichen Lebensgefühl beteiligt war, zeigt Carl Wege in seiner Studie über die Zusammenarbeit Brecht / Feuchtwanger (Carl Wege: Bertolt Brecht/Lion Feuchtwanger: Kalkutta, 4. Mai. Ein Stück Neue Sachlichkeit, München, 1988). Der eher vom Expressionismus beeinflußte Bronnen wandte sich, nach kurzer Liebäugelei mit sozialistischen Ideen, schnell der politisch Rechten zu, und sein Bekenntnis zum radikalen Nationalismus datiert aus der Entstehungszeit des la Marr-Romans, den er selbst als «antibolschewistisches Buch»im Vorwort ankündigte. Mit Sicherheit ist es kein Zufall, daß Brecht ein biographisches Bindeglied zwischen beiden Autoren darstellte.

45. - «Die Petroleuminseln» stammen nach jüngsten Hinweisen von Wege, der sich auf Martha Feuchtwanger bezieht, aus dem Jahr 1926; die Uraufführung des Stückes fand 1927 statt. Der La Marr-Roman entstand ebenfalls 1926, nachdem Bronnen «ein kleines Manuskriptbündel» mit Notizen und Tagebuch-Aufzeichnungen in die Hände gespielt wurde (vg. arnolt bronnen gibt zu Protokoll, S. 152). Der Roman erscheint 1927 im Vorabdruck in der mondänen Zeitschrift «Die Dame» und schließlich bei Rowohlt als Buch.

46. - Auf die Rekonstruktion der ökonomischen Handlung des Stückes muß in diesem Referat verzichtet werden.

47. - Zu meinem Stück «Die Petroleuminseln», in Weltbühne 23/1927, S. 602.

48. - Symptomatisch für die oberflächliche Rezeption sind die kurzschlüssigen MotivAnalogien. Das Finale des Maultasch-Romans stellt die Protagonistin aber in jeder Hinsicht als Verliererin aus; Gray dagegen verwandelt ihre moralische Niederlage (und ihre Niederlage als Frau) in einen beträchtlichen politischen und ökonomischen Sieg. Gray als die konsequente Personifikation des Prinzips Sachlichkeit hat im Stück gelernt, daß die «warme, gewachsene Mütterlichkeit, die die Maultasch «in ihre Regierung senken will» (Lion Feuchtwanger: Die häßliche Herzogin Margarete Maultasch, Frankfurt, 1984, S. 130), diesem Prinzip diametral gegenübersteht.

49. - Vgl. Hans Kaufmann, Krise und Wandlungen der deutschen Literatur von Wedekind bis Feuchtwanger, Berlin, 1966, S. 461.

50. - Wolf gang Jeske/Peter Zahn, Lion Feuchtwanger oder arge Weg der Erkenntnis, Stuttgart, 1984, S. 97.

51. - Die Romane der Weltwirtschaftskrise werden schließlich zeigen, daß die ökonomische Liquidation der Männer durch diese immer auch als sexuelle Impotenz imagi-niert wird. 
52. - Sowohl Feuchtwanger als auch Bronnen waren beide nicht in Amerika gewesen. Über die Neigung der Intellektuellen, Amerika zur Projektionsfläche deutscher Erfahrungen und Probleme zu machen vgl. Erhard Schütz, Kritik der literarischen Reportage, München, 1977, S. $18 \mathrm{f}$.

53. - Ein von Feuchtwanger durchaus ironischer Seitenhieb auf den Männlichkeitskult der Neuen Sachlichkeit.

54. - Vgl. Anm. 38, S. 54.

55. - Zur ideologischen Vergesellschaftung des Weiblichen im Film sowie eine ausführliche Analyse des Bronnen-Romans vgl. Ulrike Baureithel, «Die letzte tolle Karte im Männerspiel». Über Arnolt Bronnens Roman «Film und Leben Barbara la Marr» mit einem Ausblick auf die Septembernovelle, in Literatur für Leser 3/90, S. 141-54.

56. - Arnolt Bronnen, «Die weibliche Kriegsgeneration», in Der Vormarsch 2/1929, S. 369-372; neuerdings in Arnolt Bronnen, Sabotage der Jugend. Kleine Arbeiten 1922-1934, Innsbruck, 1989, S. lolff.

57. - Ebda, S. 370.

58. - Vgl. hierzu den Roman Barbara Bronnens «Die Tochter, München» 1980, S. 166 und 286, außerdem Hildegard Bronnen, «Unteilbar und untrennbar» sowie Renate Bronnen, «Kleine Bertalottin» in Ulrike Edschmid, Jenseits des Schreibtischs, Neuwied, 1990.

59. - Reinhold Jaretzky, «Lion Feuchtwanger und die Neue Sachlichkeit», in German Studies in India 10/1986, S. 60.

60. - Vgl. Anm. 34, S. 28.

61. - Bronnens fünf Finger, in Literatrische Welt 1/2, 1926.

62. - CG. Jung, «Das Seelenproblem des modernen Menschen» (1928), in Ders., Seelenprobleme der Gegenwart, Ölten, 1973, S. 279-301.

63. - Friedbert Aspetsberger, «Jugendbewegung, Mutterleibsphantasien und Kristallandschaften», in Le texte et l'idée, 3/1988, S. 95-120, hier: S. 118.

64. -

- Marianne Schuller, «Die Nachtseite der Humanswissenschaft», in Gabriele Dietze (Hg.), Die Überwindung der Sprachlosigkeit, Neuwied, 1979, S. 31-50, hier S. 40.

65. - Bernard v. Brentano, «Leben einer Schauspielerin», in Weltbühne, 5/1928, S. 173.

66. - Vgl. Anm. 45, S. 92.

67. - Ursula Krechel, «Linksseitig kunstseidig. Dame, Girl und Frau», in Anm. 35, S. 97-117, hier: S. 102.

68. - Siegried Kracauer, Ginster. Von ihm selbst geschrieben, Frankfurt, 1973, S. 53.

69. - Ebda., S. 131; das Tarnkappenmotiv führt Kracauer ebenfalls für seinen Helden in den Roman ein, vgl. S. 112.

\section{RÉSUMÉS}

Der Beitrag kontrastiert zunächst den zeitgenössischen publizistischen Diskurs über den Sinnverlust der männlichen Existenz mit dem Versuch der neusachlichen Autoren, die von ihnen vertretene literarische Strömung als «männliche Literatur» zu stilisieren. An zwei exemplarischen Texten, an Lion Feuchtwangers «Petroleuminseln» und Arnolt Bronnens «Film und Leben Barbara la Marr» wird gezeigt, daß es sich bei der eingeforderten «Neutralität» der Neuen Sachlichkeit um eine Ausgleichsstrategie einer um ihre Identität gebrachten 
Männergeneration handelt. Das dabei auftretende weibliche Personal verweist dabei nicht nur auf die imaginierten männlichen Unterlegenheitsängste, sondern konterkariert auch die von der Neuen Sachlichkeit proklamierte Geschichtslosigkeit der Epoche.

Cette contribution veut d'abord faire apparaître le contraste entre le discours sur la perte de sens affectant l'existence masculine et, d'autre part, la tentative des auteurs « néo-objectivistes » de donner à leur courant littéraire un style de «littérature masculine ». Deux textes exemplaires à cet égard, Les îles du Pétrole de Lion Feuchtwanger et Barbara la Marr. Vie et cinéma de Arnolt Bronnen permettent de montrer que l'exigence de «neutralité » qu'articule la « Nouvelle Objectivité » constitue en fait une stratégie de compensation mise en œuvre par une génération d'hommes à qui on a ôté leur identité. Les figures féminines ne sont pas seulement des indicateurs révélant les angoisses liées au sentiment d'infériorité dans l'imaginaire masculin; elles vont également à rencontre de la conception du présent proclamée par la «Nouvelle Objectivité », celle d'une époque qui échappe à l'Histoire.

\section{AUTEUR}

ULRIKE BAUREITHEL

Berlin 